J. KSIAM Vol.16, No.4, 217-223, 2012

\title{
SUBNORMAL WEIGHTED SHIFTS WHOSE MOMENT MEASURES HAVE POSITIVE MASS AT THE ORIGIN
}

\author{
MI RYEONG LEE ${ }^{\dagger}$ AND KYUNG MI KIM
}

Department of Mathematics, Kyungpook National University, Daegu, 702-701 Korea

E-mail address: leemr@knu.ac.kr; kkm907@hanmail.net

\begin{abstract}
In this note we examine the effects on subnormality of adding a new weight or changing some weights for a given subnormal weighted shift. We consider a subnormal weighted shift with a positive point mass at the origin by means of continuous functions. Finally, we introduce some methods for evaluating point mass at the origin about moment measures associated with weighted shifts.
\end{abstract}

\section{INTRODUCTION}

Let $\mathcal{H}$ be a separable, infinite dimensional, complex Hilbert space and let $\mathcal{L}(\mathcal{H})$ denote the algebra of all bounded linear operators on $\mathcal{H}$. For an operator $T$ in $\mathcal{L}(\mathcal{H}), T$ is called normal if $T^{*} T=T T^{*}$ and subnormal if $T$ has normal extension on some Hilbert space containing $\mathcal{H}$. Recall that, given a bounded sequence of positive real numbers $\alpha: \alpha_{0}, \alpha_{1}, \cdots$, the weighted shift $W_{\alpha}$ associated with a weight sequence $\alpha$ is an operator on $\ell^{2}\left(\mathbb{Z}_{+}\right)$defined by $W_{\alpha} e_{n}:=$ $\alpha_{n} e_{n+1}$ for all $n \geq 0$, where $\left\{e_{n}\right\}_{n=0}^{\infty}$ is the orthonormal basis for $\ell^{2}$. In particular, $W_{\alpha}$ is normal if and only if $\alpha_{n}=0$ for all $(n \geq 0)$. And we note that for a subnormal weighted shift $W_{\alpha}$ with $\alpha_{n}=\alpha_{n+1}$ for some $n \in \mathbb{N} \cup\{0\}$, subnormality of $W_{\alpha}$ immediately forces the weight $\alpha$ to be flat, that is, $\alpha_{1}=\alpha_{2}=\cdots$ ([7]). So we may assume that the weight sequence $\alpha=\left\{\alpha_{n}\right\}_{n=0}^{\infty}$ for a subnormal weighted shift $W_{\alpha}$ satisfies a strictly positive sequence converging to 1 and $\alpha_{n}<\alpha_{n+1}(n \geq 0)$ to escape the trivial case.

It is well known for a description of subnormality for weighted shifts, called Berger's Theorem ([3]) that $W_{\alpha}$ is subnormal if and only if there exists a Borel probability measure $\mu$ supported in $\left[0,\left\|W_{\alpha}\right\|^{2}\right]$ such that $\beta_{n}^{2}=\int_{\left[0,\left\|W_{\alpha}\right\|^{2}\right]} t^{n} d \mu(n \geq 0)$, where the moments of $W_{\alpha}$ are defined by $\beta_{0}:=1$ and $\beta_{n}:=\beta_{n-1} \alpha_{n-1}(n \geq 1)$. In such case, we call the measure $\mu, m o-$ ment measure for the subnormal shift $W_{\alpha}$. Recall that a weighted shift with weights $\left\{\alpha_{n}\right\}_{n=0}^{\infty}$ has a subnormal backward extension if for some positive number $\alpha_{-1}$, the weighted shift with

Received by the editors January 26 2012; Accepted November 192012.

2010 Mathematics Subject Classification. 47B20, 47B37, 44A60.

Key words and phrases. subnormal, weighted shift operator, moment measure.

The first author was supported by Basic Science Research Program through the National Research Foundation of Korea(NRF) funded by the Ministry of Education, Science and Technology(2010-0025830).

${ }^{\dagger}$ Corresponding author. 
weights $\left\{\alpha_{n-1}\right\}_{n=0}^{\infty}$ is subnormal (cf. [4], [5]). In [1], R. Curto proved that a backward extension of a subnormal weighted shift fails to be subnormal whenever the associated probability measure has a positive mass at the origin. In [4], Hoover-Jung-Lambert have studied relationship between a subnormal weighted shift and its corresponding moment measures.

This note consists of three sections. In Section 2, we construct a subnormal weighted shift and a Borel probability measure with a positive point mass using a continuous function satisfying some integration formula. In Section 3, we obtain some formulas to get the positive value at the origin of the moment measure of a subnormal weighted shift. And we give some methods and concrete examples for evaluating such point mass at the origin.

Some of calculations in Section 2 and 3 are obtained throughout computer experiments using software tool Mathematica [8].

\section{CONSTRUCTION OF SUBNORMALITY}

Before we begin our work, we introduce a criterion for subnormal extensions of weighted shifts.

Proposition 2.1. ([1]) Let $W_{\alpha}$ be a weighted shift with a weight sequence $\alpha=\left\{\alpha_{n}\right\}_{n=0}^{\infty}$ whose restriction to the subspace spanned by $\left\{e_{1}, e_{2}, \ldots\right\}$ is subnormal with associated measure $\mu$. Then $W_{\alpha}$ is subnormal if and only if

$$
\frac{1}{t} \in L^{1}(\mu) \text { and } \alpha_{0}^{2} \leq\left(\left\|\frac{1}{t}\right\|_{L^{1}(\mu)}\right)^{-1} .
$$

In particular, $W_{\alpha}$ is never subnormal when $\mu(\{0\})>0$.

Now we introduce some notations and terminology in [4]. For a weighted shift $W_{\alpha}$ with a weight sequence $\alpha=\left\{\alpha_{n}\right\}_{n=0}^{\infty}$, we denote $\left.W_{\alpha}\right|_{\mathcal{P}_{k}}$ for the restriction of $W_{\alpha}$ to the subspace $\mathcal{P}_{k}:=\vee_{i \geq k}\left\{e_{i}\right\}$. Consider a subnormal weighted shift $W_{\alpha}$ with a weight $\alpha=\left\{\alpha_{n}\right\}_{n=0}^{\infty}$ and the corresponding measure $\mu$. We write $\omega$ for a probability moment measure with the restriction $\left.W_{\alpha}\right|_{\mathcal{P}_{1}}$. Then there exists a real number $a$ with $0<a \leq 1$ such that $\mu=a \delta_{0}+\omega$ and $\omega(\{0\})=0$, where $\delta_{0}$ denotes the Kronecker function.

Let us fix an integer $N \geq 1$, and define the restriction sequence $\alpha(N)$ of $\alpha$ by $\alpha(N)$ : $\alpha_{N}, \alpha_{N+1}, \ldots$. Then the corresponding weighted shift $W_{\alpha(N)}$ is unitarily equivalent to $\left.W_{\alpha}\right|_{\mathcal{P}_{N}}$. Since this is the restriction of a subnormal operator to an invariant subspace, $W_{\alpha(N)}$ is itself a subnormal weighted shift (with norm 1). Let $\mu_{N}$ be its associated probability measure and write $\mu_{N}=a_{N} \delta_{0}+w_{N}$. Then we can have that the following relationships for these measures ([4]):

$$
t d \mu_{N}=\frac{t^{N+1}}{\beta_{N}^{2}} d \mu \quad \text { and } \quad d w_{N}=\frac{t^{N}}{\beta_{N}^{2}} d \mu=\frac{t^{N}}{\beta_{N}^{2}} d w .
$$

Proposition 2.2. Suppose that $W_{\alpha}$ is a subnormal weighted shift with a weight sequence $\alpha=$ $\left\{\alpha_{n}\right\}_{n=0}^{\infty}$ and the associated measure $\mu$. Let $\widehat{\alpha}(k, x): x, \alpha_{k}, \alpha_{k+1}, \cdots$ be a weight sequence by prefixing a positive real variable $x$ to the restriction sequence $\alpha(k)$ of $\alpha$ and let $W_{\widehat{\alpha}(k, x)}$ be an associated weighted shift with a weight $\widehat{\alpha}(k, x)(k \geq 0)$. 
(i) For $k=0, W_{\alpha(0, x)}$ is subnormal if and only if

$$
x \leq \frac{\alpha_{0}}{\sqrt{\omega([0,1])}}
$$

where $\mu=a \delta_{0}+\omega$ with $\omega(\{0\})=0$. In particular, if $\mu(\{0\})=a>0$, then $\omega([0,1])=1-a$, and so $W_{\alpha(x)}$ is subnormal if and only if

$$
0<x \leq \frac{\alpha_{0}}{\sqrt{1-a}}
$$

(ii) For $k \geq 1, W_{\widehat{\alpha}(k, x)}$ is subnormal if and only if there exists a Borel probability measure $\mu_{(k ; x)}$ corresponding to $W_{\widehat{\alpha}(k, x)}$ such that

$$
d \mu_{(k ; x)}=\left[1-\left(\frac{x}{\alpha_{k-1}}\right)^{2}\right] d \delta_{0}+\frac{x^{2}}{\beta_{k}^{2}} t^{k-1} d \mu .
$$

Proof. (i) See [4, Theorem 3.3].

(ii) We show the existence of a Borel probability measure on $[0,1]$ for a backstep extension $W_{\widehat{\alpha}(k, x)}$ of restriction weighted shift $\left.W_{\alpha}\right|_{\mathcal{P}_{k}}$ for $k \geq 1$. Suppose that $W_{\widehat{\alpha}(k, x)}$ is subnormal. Then there exists a probability measure $\widehat{\mu}_{(k ; x)}$ on $[0,1]$ such that

$$
x^{2} \alpha_{k}^{2} \alpha_{k+1}^{2} \cdots \alpha_{k+n-2}^{2}=\int_{[0,1]} t^{n} d \widehat{\mu}_{(k ; x)} .
$$

Using (2.1) and the definition of the sequence $\left\{\beta_{n}\right\}$, we can obtain that

$$
\begin{aligned}
x^{2} \int_{[0,1]} t^{n} d \mu_{k} & =x^{2} \int_{0}^{1} t^{n-1} \frac{t^{k+1}}{\beta_{k}^{2}} d \mu \\
& =\frac{x^{2}}{\beta_{k}^{2}} \beta_{n+k}^{2}=x^{2} \alpha_{k}^{2} \cdots \alpha_{k+n-1}^{2},
\end{aligned}
$$

which is equivalent to

$$
t d \widehat{\mu}_{(k ; x)}=x^{2} d \mu_{k}
$$

Hence we have that

$$
d \widehat{\mu}_{(k ; x)}=\frac{x^{2}}{\beta_{k}^{2}} t^{k-1} d \mu+a_{k} d \delta_{0}
$$

for some $0 \leq a_{k}<1$. Also, we can obtain that

$$
\widehat{\mu}_{(k ; x)}([0,1])=\frac{x^{2}}{\beta_{k}^{2}} \int_{(0,1]} t^{k-1} d \mu+a_{k} \delta_{0}(\{0\})=1
$$

and by the definition of sequence $\beta_{k}^{2}$, we obtain that

$$
\widehat{\mu}_{(k ; x)}(\sigma)=\frac{x^{2}}{\beta_{k}^{2}} \cdot \mu(\sigma)+\left(1-\frac{x^{2}}{\alpha_{k-1}^{2}}\right) \delta_{0}(\sigma)
$$

for any Borel subset $\sigma$ in $[0,1]$. 
Until now we have discussed a point mass at the origin from the given subnormal weighted shift. Conversely from the given a point mass, we now discuss a subnormal weighted shift via a continuous function on $[0,1]$ using a integration formula below.

Theorem 2.3. For $0<a<1$, let $\varphi$ be a continuous function on $[0,1]$ with $\varphi(0)=0$ and $\varphi(t) \geq 0$ on $(0,1]$ such that

$$
\int_{0}^{1} \varphi(t) d t=1-a .
$$

Then there exists a sequence $\alpha:=\left\{\alpha_{n}\right\}_{n=0}^{\infty}$ such that the corresponding weighted shift is subnormal whose associated measure $\mu$ on $[0,1]$ satisfies

$$
d \mu=a d \delta_{0}+\varphi d t
$$

Proof. First we define a sequence $\left\{\beta_{n}\right\}_{n=0}^{\infty}$ by

$$
\beta_{n}:=\left\{\begin{array}{cl}
1 & \text { if } n=0, \\
\sqrt{\int_{0}^{1} t^{n} \varphi(t) d t} & \text { if } n \geq 1 .
\end{array}\right.
$$

Define a measure $\mu$ on $[0,1]$ such that

$$
\mu(\sigma)=a \cdot \delta_{0}(\sigma)+\int_{\sigma} \varphi(t) d t
$$

for any Borel subset $\sigma$ in $[0,1]$. Observe that, for $n \geq 1$, by (2.2) we have

$$
\begin{aligned}
\int_{[0,1]} t^{n} d \mu & =\int_{\{0\}} t^{n} d \mu+\int_{(0,1]} t^{n} d \mu=0+\int_{(0,1]} t^{n} \varphi(t) d t \\
& =\int_{[0,1]} t^{n} \varphi(t) d t=\beta_{n}^{2} .
\end{aligned}
$$

Hence, for all $n \geq 0$, we have $\beta_{n}^{2}=\int_{[0,1]} t^{n} d \mu$. Since $\mu$ is a probability measure and $\left\{\beta_{n}\right\}_{n=0}^{\infty}$ is the moment sequence, the measure $\mu$ induces a subnormal weighted shift associated with the weight sequence $\left\{\frac{\beta_{n+1}}{\beta_{n}}\right\}_{n=0}^{\infty}$.

Example 2.4. In Theorem 2.3, we consider $\varphi(t)=6(1-a)\left(t-t^{2}\right)$ with $0<a<1$. Then we obtain the weight sequence $\alpha=\left\{\alpha_{n}\right\}_{n=0}^{\infty}$ for subnormality of weighted shift with

$$
\alpha: \alpha_{0}=\sqrt{\frac{1-a}{2}}, \alpha_{n}=\sqrt{\frac{n+2}{n+4}} \quad(n \geq 1) .
$$

Indeed, for $n \geq 1$,

$$
\beta_{n}^{2}=\int_{0}^{1} t^{n} \varphi(t) d t=\frac{6(1-a)}{(n+2)(n+3)} .
$$

Put $\alpha_{n}=\frac{\beta_{n+1}}{\beta_{n}}$ for $n \geq 1$. Then $\alpha_{n}=\sqrt{\frac{n+2}{n+4}}(n \geq 1)$. From $\alpha_{0}=\beta_{1}=\sqrt{\frac{1-a}{2}}$, we can have a weight sequence $\alpha=\left\{\alpha_{n}\right\}_{n=0}^{\infty}$ associated the subnormal weighted shift. 
Example 2.5. In Theorem 2.3, if we take $\varphi(t)=\sin \frac{\pi}{2} t(0 \leq t \leq 1)$ and define a sequence $\left\{\beta_{n}\right\}_{n=0}^{\infty}$ as

$$
\beta_{n}^{2}:=\left\{\begin{array}{cl}
1 & (n=0), \\
\int_{0}^{1} t^{n} \sin \frac{\pi}{2} t d t & (n \geq 1),
\end{array}\right.
$$

then we have $a=1-\frac{2}{\pi}$. Put $\alpha_{n}:=\frac{\beta_{n+1}}{\beta_{n}}(n \geq 0)$. Hence we may obtain easily a weight sequence $\alpha:=\left\{\alpha_{n}\right\}_{n=0}^{\infty}$ with

$$
\begin{aligned}
& \alpha_{0}=\frac{\pi}{2}, \alpha_{1}=\sqrt{\frac{2(\pi-2)}{\pi}}, \alpha_{2}=\sqrt{\frac{3\left(\pi^{2}-8\right)}{2 \pi(\pi-2)}}, \\
& \alpha_{3}=2 \sqrt{\frac{48-24 \pi+\pi^{3}}{3 \pi\left(\pi^{2}-8\right)}}, \alpha_{4}=\frac{1}{2} \sqrt{\frac{5\left(384-48 \pi^{2}+\pi^{4}\right)}{\pi\left(\pi^{3}-24 \pi+48\right)}}, \ldots .
\end{aligned}
$$

Moreover, the probability measure $d \mu=\left(1-\frac{2}{\pi}\right) d \delta_{0}+\sin \frac{\pi}{2} t d t$ on $[0,1]$ determines the subnormality of the weighted shift $W_{\alpha}$.

\section{COMPUTATIONS OF POINT MASS AT THE ORIGIN}

In this section, in order to compute the positivity of moment measure at the origin for a subnormal weighted shift, we introduce some fundamental formulas for evaluating point mass at the origin for the measure associated with a weighted shift.

Lemma 3.1. ([4]) Let $W_{\alpha}$ be a subnormal weighted shift with a weight sequence $\alpha$ and a moment measure $\mu$. Then

$$
\mu(\{0\})=\lim _{k \rightarrow \infty} \sum_{j=0}^{k}(-1)^{j}\left(\begin{array}{l}
k \\
j
\end{array}\right) \beta_{j}^{2}=\lim _{k \rightarrow \infty} \sum_{j=0}^{\infty} \frac{(-k)^{j}}{j !} \beta_{j}^{2}
$$

where $\beta_{0}=1$ and $\beta_{n}^{2}=\int_{0}^{1} t^{n} d \mu(n \geq 1)$.

If we use the notion of the incomplete gamma function $\Gamma(a, z):=\int_{z}^{\infty} t^{a-1} e^{-t} d t$, we can have the following simple result.

\section{Lemma 3.2.}

$$
\lim _{n \rightarrow \infty} \Gamma(0, n)=\lim _{n \rightarrow \infty} \int_{n}^{\infty} \frac{1}{t e^{t}} d t=0 .
$$

Proof. For $1 \leq n \leq t$, we have that $0<t^{-1} e^{-t} \leq e^{-t}$. Using definitions of improper integrals, we have $\int_{n}^{\infty} e^{-t} d t=e^{-n}$. Hence

$$
\lim _{n \rightarrow \infty} \int_{n}^{\infty} e^{-t} d t=0 .
$$

This completes our result. 
Example 3.3. Consider a weight sequence $\alpha: \alpha_{n}=\sqrt{\frac{(n+1)\left(2^{n+2}-1\right)}{2(n+2)\left(2^{n+1}-1\right)}}(n \geq 0)$. Let $d \mu=$ $2 \cdot \chi_{\left[\frac{1}{2}, 1\right]} d t$. Since

$$
\beta_{n}^{2}=\int_{0}^{1} t^{n} d \mu=2 \int_{\frac{1}{2}}^{1} t^{n} d t=\frac{2}{n+1}\left(1-\frac{1}{2^{n+1}}\right),(n \geq 1)
$$

and

$$
\beta_{0}=\int_{0}^{1} d \mu=1,
$$

the weighted shift $W_{\alpha}$ is a subnormal with the associated measure $\mu$. Let a weight sequence $\widehat{\alpha}(x)(\equiv \widehat{\alpha}(0, x)): x, \alpha_{0}, \alpha_{1}, \alpha_{2}, \ldots$ be a backward extension of $\alpha$. Hence $W_{\widehat{\alpha}(x)}$ is subnormal if and only if

$$
x^{2} \int_{0}^{1} \frac{1}{t} d \mu=2 x^{2} \int_{\frac{1}{2}}^{1} \frac{1}{t} d t=2 x^{2} \ln 2 \leq 1,
$$

which is equivalent to $0<x \leq \sqrt{\frac{1}{2 \ln 2}}$. By Lemma 3.1, we obtain

$$
\begin{aligned}
\mu(\{0\}) & =1-\lim _{n \rightarrow \infty} \sum_{i=1}^{n}(-1)^{i+1}\left(\begin{array}{c}
n \\
i
\end{array}\right) \beta_{i}^{2} \\
& =1-\lim _{n \rightarrow \infty} \frac{n+1-2^{-n}}{n+1}=0 .
\end{aligned}
$$

Now we consider $\alpha^{\prime}\left(\equiv \widehat{\alpha}\left(1, \alpha_{0}^{\prime}\right)\right)$ with $\alpha_{0}^{\prime}=\sqrt{\frac{1}{4 \ln 2}}$ and $\alpha_{n}^{\prime}=\alpha_{n}(n \geq 1)$. We recall that the associated moment sequence $\left\{\beta_{n}^{\prime 2}\right\}_{n=0}^{\infty}$ is given by $\beta_{0}^{\prime}=1$ and $\beta_{n}^{\prime}=\alpha_{n-1}^{\prime} \cdot \beta_{n-1}^{\prime}$ for $n \geq 1$. From the Proposition 2.2, we take an corresponding probability measure $\mu^{\prime}:=\mu_{\left(1 ; \alpha_{0}^{\prime}\right)}$ with $W_{\alpha^{\prime}}$. By simple computations, we can have that for all $n \geq 1$,

$$
\beta_{n}^{\prime 2}=\frac{2^{n+1}-1}{2^{n}(n+1) 3 \ln 2} \leq \frac{2}{3(n+1) \ln 2} .
$$

We use the binomial theorem for the calculation $\int_{0}^{1 / 2}(1-x)^{n} d x$, we can obtain

$$
\sum_{i=1}^{n}(-1)^{i+1}\left(\begin{array}{c}
n \\
i
\end{array}\right)\left(\frac{2}{i+1}-\frac{2^{i+1}-1}{(i+1) 2^{i}}\right)=\frac{2^{n}(n-1)+1}{(n+1) 2^{n}} \geq 0 \text { for } n \geq 1,
$$

which deduces the following result:

$$
\sum_{i=1}^{n}(-1)^{i+1}\left(\begin{array}{l}
n \\
i
\end{array}\right){\beta_{i}^{\prime}}^{2} \leq \frac{2}{3 \ln 2} \sum_{i=1}^{n}(-1)^{i+1}\left(\begin{array}{l}
n \\
i
\end{array}\right) \frac{1}{i+1}
$$

for all $n \geq 1$. Also we calculate the value of $\int_{0}^{1}(1-x)^{n} d x$ via the binomial theorem, we can obtain that

$$
\frac{1}{2}\left(\begin{array}{l}
n \\
1
\end{array}\right)-\frac{1}{3}\left(\begin{array}{l}
n \\
2
\end{array}\right)+\cdots+(-1)^{n+1} \frac{1}{n+1}\left(\begin{array}{l}
n \\
n
\end{array}\right)=\frac{n}{n+1}
$$


for all $n \geq 1$. To show the positivity of the point mass $\mu^{\prime}(\{0\})$ in Lemma 3.1, using (3.1) and (3.2), we can have that

$$
\begin{aligned}
\mu^{\prime}(\{0\}) & =\lim _{n \rightarrow \infty} \sum_{i=0}^{n}(-1)^{i}\left(\begin{array}{c}
n \\
i
\end{array}\right) \beta_{i}^{\prime 2}=1-\lim _{n \rightarrow \infty} \sum_{i=1}^{n}(-1)^{i+1}\left(\begin{array}{c}
n \\
i
\end{array}\right) \beta_{i}^{\prime 2} \\
& \geq 1-\lim _{n \rightarrow \infty} \frac{2 n}{3(n+1) \ln 2}=\frac{3 \ln 2-2}{3 \ln 2}
\end{aligned}
$$

which guarantees that $\mu^{\prime}(\{0\})>0$.

Example 3.4. For the weight sequence $\alpha$ in Example 3.3, if we consider $\alpha^{\prime} \equiv \widehat{\alpha}\left(2, \alpha_{0}^{\prime}\right)$ with $\alpha_{0}^{\prime}=\sqrt{\frac{1}{2 \ln 2}}$ and $\alpha_{n}^{\prime}=\alpha_{n+1}(n \geq 1)$. And applying Lemma 3.1 and 3.2 with $\alpha^{\prime}$ and we have the corresponding probability measure $\mu^{\prime} \equiv \mu_{\left(2 ; \alpha_{0}^{\prime}\right)}$, we can have that

$$
\begin{aligned}
\mu^{\prime}(\{0\}) & =\lim _{n \rightarrow \infty} \sum_{i=0}^{\infty} \frac{(-n)^{i}}{i !}\left(\beta_{i}^{\prime}\right)^{2} \\
& =\frac{1}{\ln 2} \lim _{n \rightarrow \infty}\left(\Gamma\left(0, \frac{n}{2}\right)-\Gamma(0, n)\right)=0 .
\end{aligned}
$$

Now to show the positivity of the measure at the origin as in Example 3.3, we have to take a real number $a$ satisfying $a>2 \ln 2$ for a new weight sequence $\alpha^{\prime \prime} \equiv \widehat{\alpha}(2, a)$ with $\alpha_{0}^{\prime \prime}=\sqrt{\frac{1}{a}}$ and $\alpha_{n}^{\prime \prime}=\alpha_{n+1}(n \geq 1)$ and use the similar methods in Example 3.3. We leave some computations to interested readers.

\section{REFERENCES}

[1] R. Curto, Quadratically hyponormal weighted shifts , Integral Equations and Operator Theory 13(1990), 4966.

[2] R. Curto and L. Fialkow, Recursively generated weighted shifts and the subnormal completion problem, I, Integral Equations and Operator Theory 17(1993), 202-246.

[3] P. Halmos, Ten problems in Hilbert space, Bull. Amer. Math. Soc. 76(1970), 887-933.

[4] T. Hoover, I. B. Jung and A. Lambert, Moment sequences and backward extensions of subnormal weighted shifts, J. Austral. Math. Soc. 73(2002), 27-36.

[5] Z. Jabłonski, I. B. Jung and J. Stochel, Backward extensions of hyperexpansive operators, Studia Math. 173(2006), 233-257.

[6] A. Lambert, Subnormality and weighted shifts, J. London Math. Soc. 14(1976), 476-480.

[7] J. Stampfli, Which weighted shifts are subnormal, Pacific J. Math. 17(1966), 367-379.

[8] Wolfram Research, Inc. Mathematica, Version 8.0, Wolfram Research Inc., Champaign, IL, 2010. 